\title{
Full Mouth Rehabilitation of Severely Collapsed Vertical Dimension in a Geriatric Patient with Extra Coronal Attachment
}

\author{
Vaishnavi Rajaraman¹, Deepak Nallaswamy Veeraiyan², Suresh Venugopalan³, Subha M4
}

\begin{abstract}
${ }^{1}$ Department of Prosthodontics and Implantology, Saveetha Dental College and Hospital, Saveetha Institute of Medical and Technical Sciences, Chennai, Tamil Nadu, India. ${ }^{2}$ Department of Prosthodontics and Implantology, Saveetha Dental College and Hospital, Saveetha Institute of Medical and Technical Sciences, Chennai, Tamil Nadu, India. ${ }^{3}$ Department of Prosthodontics and Implantology, Saveetha Dental College and Hospital, Saveetha Institute of Medical and Technical Sciences, Chennai, Tamil Nadu, India. ${ }^{4}$ Department of Oral Medicine and Radiology, Saveetha Dental College and Hospital, Saveetha Institute of Medical and Technical Sciences, Chennai, Tamil Nadu, India.
\end{abstract}

\section{PRESENTATION OF CASE}

An 80 years old male patient came to the Department of Prosthodontics and Implantology, Saveetha Dental College with the chief complaint of inability to chew food and unaesthetic smile. Patient had a medical history of hypertension of 10 years and diabetes mellitus of 6 years. He was under medication for the same. Clinical examination of the oral cavity revealed multiple missing, few extensively decayed teeth and absolute vertical collapse.

The patient had severe attrition of maxillary occlusal and mandibular occlusal aspects and a massive deep bite scenario with completely collapsed vertical dimension. Patient had no symptoms of TMJ dysfunction or any signs and symptoms of acute infection. The teeth missing were anterior teeth in the region $11,12,21,23$, posterior teeth in region $44,45,46,47$ and 35,36 . Severe bone atrophy was observed in the anterior maxillary and posterior mandibular area of the oral cavity. Smile line of the patient was Class 3 (average smile line), according to Liebart classification. ${ }^{1}$ Teeth requiring endodontic intervention, were diagnosed in relation to $26,33,34,43$. These teeth had severe attrition with exposure of pulp chambers and also had inadequate crown structure. Intentional endodontic therapy with lateral condensation obturation technique was planned for these teeth. ${ }^{2,3}$ The most challenging teeth for endodontic therapy was 26 , as it involved decay below the CEJ which was saved for preserving it as a terminal abutment. $2,4,5$

The diagnosis and treatment protocol of tooth wear is intricate and complex, and a multidisciplinary approach is required for its management. One of the most intellectually and technically challenging tasks facing a prosthodontist is planning and executing the rehabilitation of a severely decimated occlusion. To obtain effective and predictable outcome, appropriate diagnosis and elaborate treatment protocol is essential. The goal in full mouth rehabilitation, understanding of the aetiology, education the patient about prevention and approach for the preservation of the remaining tooth structure.
Corresponding Author:

Dr. Vaishnavi Rajaraman.

162, Poonamallee High Road,

Chennai-600077, Tamil Nadu, India.

E-mail:drvaish.sav@gmail.com

DOI: $10.14260 / \mathrm{jemds} / 2020 / 605$

How to Cite This Article:

Rajaraman V, Veeraiyan DN, Venugopalan $S$, et al. Full mouth rehabilitation of severely collapsed vertical dimension in a geriatric patient with extra coronal attachment. J Evolution Med Dent Sci 2020;9(37):27782782, DOI: $10.14260 /$ jemds/2020/605

Submission 11-06-2020,

Peer Review 05-08-2020,

Acceptance 11-08-2020,

Published 14-09-2020.

Copyright (C) 2020 JEMDS. This is an open access article distributed under Creative Commons Attribution License [Attribution 4.0 International (CC BY 4.0)] 
This case report describes the full mouth rehabilitation of severely collapsed vertical dimension of a patient who underwent periodontal and endodontic intervention which was followed by full veneer porcelain fused to metal fixed prosthesis for the complete maxillary arch and anterior and left posterior mandibular arch with extracoronal attachments supported cast partial denture framework for the right posteriors in the mandibular arch.

\section{DISCUSSION OF MANAGEMENT}

\section{Treatment Plan}

Step 1: Preoperative Photographs (with patient's consent) (Figure 1), Diagnostic impressions with irreversible hydrocolloid (Zhermack, Tropicalgin ${ }^{\circledR}$ ), tentative facebow record

Step 2: Anterior tooth preparation and temporization (with prefabricated polymethylmethacrylate).

Step 3: Posterior tooth preparation and master impression of maxillary and mandibular arches (Zhermack Elite HD+ ${ }^{\circledR}$ ) followed by full mouth temporization (Figure 2).

Step 4: Facebow record, vertical and centric jaw relation

Step 5: Metal trial of both arches with the attachment in the lower arch and verification of jaw relation

Step 6: Ceramic bisque trial and pick up impression (Zhermack Elite $\mathrm{HD}+{ }^{\circledR}$ ) of the lower arch for the cast partial framework fabrication

Step 7: Cast partial framework trial with teeth setting and verification of occlusion

Step 8: Final cementation and insertion of prosthesis (Figure 1) followed by maxillary arch impression with irreversible hydrocolloid (Zhermack, Tropicalgin ${ }^{\circledR}$ ), for night guard fabrication and postoperative photographs with frontal smile.

Step 9: Night guard delivery after checking occlusal contacts Step 10: Follow up and panoramic radiograph after 3 months (Figure $3 \& 4$ ).

Implant supported fixed prosthesis was recommended to the patient for the partially edentulous portions of the maxillary and mandibular arches but was refused by the patient due to various factors like age, medical history and invasiveness of the procedure.6,7 Due to severe attrition and absolutely collapsed bite, full veneer fixed restoration of the remaining teeth and removable prosthesis for the distal extension was planned for long term predictability. ${ }^{8-11}$ For Mandibular posterior partially edentulous arch, a removable prosthesis was planned that involved extra coronal attachment supported cast partial denture. ${ }^{9,12-14}$ For maxillary arch, tooth supported full coverage restoration with porcelain fused to ceramics was planned. 15

After acquiring informed consent from the patient, the Dawson technique of bilateral manipulation was carried out to position the mandible in the centric relation. The patient's vertical dimension (VD) at rest and at occlusion was determined, with the assistance of factors including anatomical landmarks, facial measurements, aesthetics and phonetics by using a vernier calliper. It was then deciphered using these methods to increase the collapsed VD by $4.0 \mathrm{~mm}$.

Casts were mounted with the facebow record (UTS transfer@) and inter occlusal records using overlay occlusal rims in centric relation onto the semi adjustable articulator (STRATOS 300, Ivovlar®), at an increased VD. Diagnostic wax up was done for maxillary and mandibular occlusal rims at the pre-determined and increased VD to establish occlusion. Provisional acrylic restoration, at increased vertical dimension, were prefabricated by indirect method with the help of silicone index made using putty consistency elastomeric impression material from the diagnostic wax up. The maxillary and mandibular anterior teeth were prepared with adequate requirements and the provisional acrylic temporaries were delivered to the patient using temporary cement. The provisional restoration was adjusted in the patient's mouth by locating all excursive pathways form centric relation to an edge to edge relationship in both protrusive and lateral jaw position to establish the anterior guidance. ${ }^{16}$ The maxillary and mandibular incisal edges of each anterior teeth were located and the labial contour of these teeth were finalized and fixed. By progressively increasing the VD in two stages, till the span of the whole treatment, any change or adaptation in relation to TMJ was evaluated during this period. Occlusal stability was also checked for the same duration of time.

After their acceptability had been confirmed in the increased vertical dimension, gingival retraction was performed to expose all sub gingival and equigingival margins of the tooth preparation, prior to impression making. For gingival retraction procedure, a double cord technique was used, a \#000 first cord and a \#00 second cord, with the cords soaked in local anaesthetic solution containing adrenaline (ULTRAPAK knitted cord).

An elastomeric impression of the prepared arches was made after meticulous and conservative gingival retraction procedure with a two-step putty wash technique. The putty impression was taken first (Elite HD + Putty Soft, Regular SetOrange), followed by a light body wash impression after the removal of the \#00 cords.

The casts were prepared and mounted on a semi adjustable articulator with the help of facebow record, centric record and with the corrected provisional restoration. The centric record was taken using overlay occlusal rims over the prepared teeth. The anterior guidance which was obtained and tested in the provisional restoration, was duplicated by use of the customized anterior guide table in the semi adjustable articulator. ${ }^{16}$ The master die model with the same centric record that was used to mount the provisional restorations was used to mount in the semi adjustable articulator. Thus the articulation relates exactly to the intraorally customized anterior guide. On the right posterior mandibular arch (4th quadrant) distal to the distal most abutments, spherical castable (Rhein 83 OT Unilateral) attachments were attached using parallelometer mandrel and the corresponding housing was placed over the OT unilateral attachment. To the housing, yellow coloured connector was also attached. Wax coping assembly with OT unilateral attachment and corresponding housing joined to the connectors were invested and then casted together for precision. The rings in the denture base are colour coded to indicate different levels of retention that can be obtained and for easier removal and insertion. ${ }^{17}$ Metal trial was done and the jaw relation verified for clearance for the ceramic layering. Shade determination (VITA classic shade guide) was done according to the existing tooth and facial tone. 

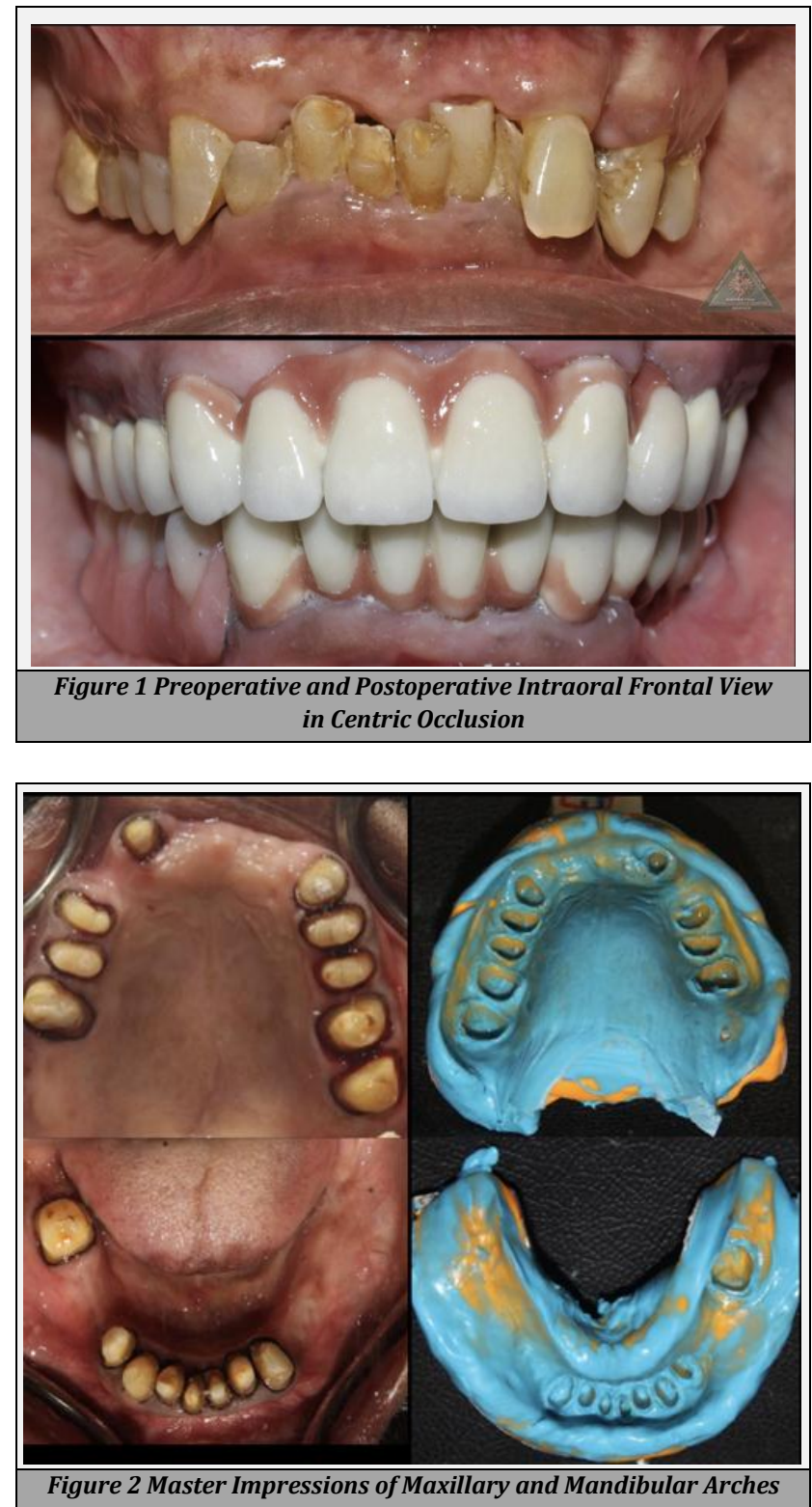

Bisque trial with the full veneer porcelain fused to metal fixed prosthesis was performed for any alterations required in occlusion or aesthetics. Final fixed prosthesis for the maxillary arch and the left posterior region of the mandibular arch was cemented with glass ionomer permanent luting cement.

After this, mandibular elastomeric pick-up impression was made for fabricating the cast partial denture framework or the lower arch. The mandibular with cast partial framework, with a precision attachment on the right side and a rest seat and circumferential clasp on the last abutment on the left side 37 over the cemented PFM (surveyed crown) was tried in the patient's mouth for retention and stability. Once the fit was confirmed, a wax trial of the lower right side with 44,45,46,47 was done and occlusion was established in centric and eccentric movements of the mandible. Finally, the cast partial denture was processed, trimmed and finished. Patient's occlusion was inspected for verification and establishment of mutually protected occlusion and maximum intercuspation in the patients recorded centric relation.

A mouth guard was given to protect the ceramic prosthesis and post-operative smile of the patient. A panoramic radiograph was taken 12 months after the prosthesis was delivered.
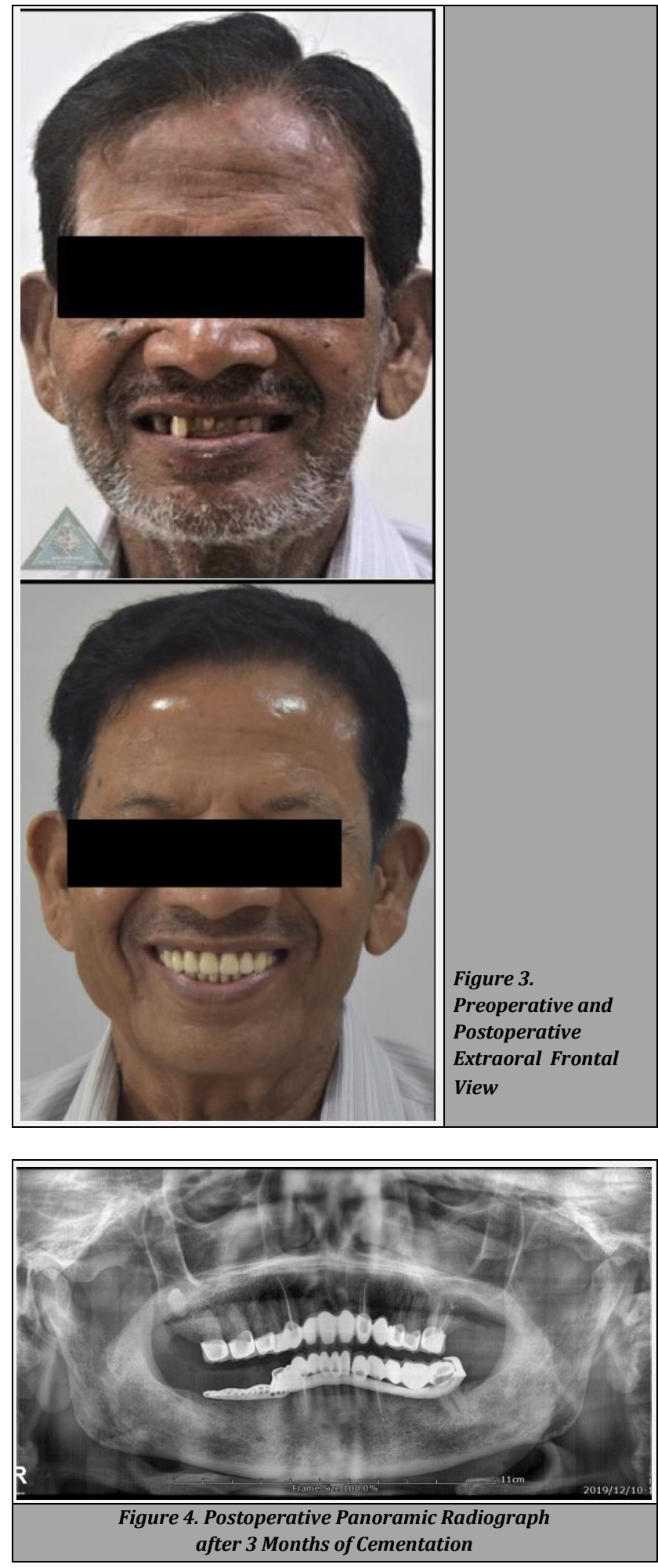

\section{DISCUSSION}

The present case report clearly depicts a successful attempt at restoring decimated dentition with severe vertical collapse by carefully planned full mouth rehabilitation with fixed prosthesis. After reviewing the results of a survey conducted among peers of dental fraternity regarding treatment of complex vertical collapse, we found that not many dentists are confident in treating such cases and the evidence supports this result. 18,19 
We dentists are well aware that non-carious surface loss of natural tooth structure is a multifactorial chain of events which encompasses the degradation of enamel and dentin along with progressive deterioration of the functional ability of the affected patients on the whole. The process of tooth wear includes the etiological factors like attrition, erosion, abrasion and abfraction which are due to non-carious reasons. ${ }^{20}$ The severe loss of occlusal vertical dimension, impaired masticatory function and unaesthetic appearance in this patient discussed in our case report, is due attrition.

The generalized tooth wear in an individual can be treated effectively by full mouth rehabilitation approach. It is of utmost importance to measure the existing difference in vertical dimension at rest and occlusion to determine the necessary post-operative end point of the prosthesis by maintaining the freeway space. In this case, a $4 \mathrm{~mm}$ increase in the vertical dimension was achieved with the assistance of parameters like anatomical landmarks, facial measurements, aesthetics and phonetics. Mutually protected type of occlusal scheme was provided to the patient in this particular case, as the maxillary and mandibular anterior teeth were periodontally sound. This was mainly done to protect the posterior teeth in all excursive movements of the mandible and consequently disocclude the anterior teeth in maximal intercuspal position when axially loaded or in centric relation. ${ }^{21}$

To provide even pressure, stress distribution and balanced support for the remaining natural teeth, extracoronal attachments were used with benefits of low maintenance and patient comfort. ${ }^{17}$ The colour coding was carefully selected for adequate retention. ${ }^{17}$ The patient was put on a recall regimen with 1, 3, 6 and 12 months follow up periods. The fixed prosthesis exhibited no signs of deterioration and the final outcome was pleasing to the patient. ${ }^{22}$ This clinical report effectively describes the systematic method and sequential treatment approach for full mouth rehabilitation of a geriatric patient with severely decimated occlusion and collapsed vertical dimension in minimal series of appointments.

\section{CONCLUSIONS}

Full mouth rehabilitation with usage of appropriate dental materials and through systematic sequential methods, increases the life span of the functioning dental health of a geriatric patient. It also assists a great deal in designing the apt dentition to accept adequate occlusal forces with minimal damage to function efficiently, for proper phonetics and pronunciation as we as aesthetically improve the facial appearance, thereby restoring his confidence.

Financial or Other Competing Interests: None.

\section{REFERENCES}

[1] Liébart MF, Fouque-Deruelle C, Santini A, et al. Smile line and periodontium visibility. Periodontal Practice Today 2004;1(1):17-25.
[2] Lin LM, Loghin S, Ricucci D. Endodontic treatment of mature teeth. In: Chugal N, Lin LM, eds. Endodontic prognosis. Springer Cham 2017:43-63.

[3] Litonjua LA, Andreana S, Bush PJ, et al. Tooth wear: attrition, erosion and abrasion. Quintessence Int 2003;34(6):435-46.

[4] Baker GJ. Dental decay and endodontic disease. In: Baker GJ, Easley J, eds. Equine dentistry. 2nd edn. Elsevier 2005:121-5.

[5] Chugal N, Lin LM. Endodontic prognosis: clinical guide for optimal treatment outcome. Springer 2016: p. 243.

[6] Khan MAI, Taleb A. Informed consent for dental treatment: a survey on dental practitioners' perception, awareness and understanding. Bangladesh J Dent Res Educ 2013;2(2):31-5.

[7] Cannavina CD, Cannavina G, Walsh TF. Effects of evidencebased treatment and consent on professional autonomy. Br Dent J 2000;188(6):302-6.

[8] Walter JD. Removable partial denture design. Br Dent Assoc 1990: p. 120.

[9] Gupta S, Rani S, Sikri A, et al. Attachment retained cast partial denture: conventional and contemporary treatment perspectives. Inter J Oral Care Res 2016;4(4):312-6.

[10] Anne G, Kadiyala KKH, Dev RRJ, et al. Full mouth rehabilitation using fixed partial denture \& cast partial denture with attachments and conventional lower denture - a case report. Inter Dent J Student Res 2017;5(1):25-8.

[11] Gupta K, Javiya P, Kumar P, et al. Rehabilitation of lost vertical dimension with cast post core and cast partial denture. BMJ Case Rep 2013;2013:bcr2013008576.

[12] Patel H, Patel K, Thummer S, et al. Use of precision attachment and cast partial denture for long-span partially edentulous mouth- a case report. Int J Appl Dent Sci 2014;1(1):22-5.

[13] Ozcelik TB, Yilmaz B. An alternative procedure for positioning a prefabricated extracoronal attachment in a removable partial denture. J Prosthetic Dentistry 2008;100:240-1.

[14] Bhatia LK, Ghosh S, Gupta H, et al. Attachment retained mandibular cast partial denture. Indian J Contemporary Dentistry 2014;2(2):107.

[15] Heschl A, Haas M, Haas J, et al. Maxillary rehabilitation of periodontally compromised patients with extensive onepiece fixed prostheses supported by natural teeth: a retrospective longitudinal study. Clin Oral Investig 2013;17(1):45-53.

[16] Kim WG, Yeom KY, Lee YS. Full mouth rehabilitation with vertical dimension increase in patient with loss of anterior guidance due to maxillary anterior teeth wear: a case report. J Korean Acad Prosthodont 2017;55(2):1719.

[17] Preiskel HW. Precision Attachments in prosthodontics: the applications of intracoronal and extracoronal attachments. Vol. 1. Quintessence Publishing Company 1984: p. 318.

[18] Yiu CKY, McGrath C, Bridges S. et al. Self- perceived preparedness for dental practice amongst graduates of the University of Hong Kong's integrated PBL dental curriculum. Euro J Dent Educ 2012;16(1):e96-e105. 
[19] Rajaraman V, Velayudhan A. Full mouth fixed rehabilitation of a young adult with ectodermal dysplasia: making mountain out of a molehill in the literal sense. J Dent Oral Sci 2020;2(2):1-2.

[20] Goldstein RE, Curtis JW, Farley BA, et al. Abfraction, abrasion, attrition, and erosion. In: Goldstein RE, Chu SJ, Lee EA, eds. Ronald E Goldstein's esthetics in dentistry. Wiley-Blackwell 2018:692-719.
[21] Rinchuse DJ, Kandasamy S, Sciote J. A contemporary and evidence- based view of canine protected occlusion. Am J Orthod Dentofacial Orthop 2007;132(1):90-102.

[22] Rajaraman V, Abraham A, Dhanraj M, et al. Symmetry of gingival zenith: defining esthetic outcome. Drug Invention Today 2018;10(5):800-4. 